\title{
ON A COUNTING FORMULA OF DJOKOVIĆ FOR ELEMENTS OF FINITE ORDER IN COMPACT LIE GROUPS
}

\author{
F. DESTREMPES AND A. PIANZOLA \\ (Communicated by Roe W. Goodman)
}

\begin{abstract}
Given a compact connected simple Lie group $\mathfrak{G}$ and a positive integer $N$ relatively prime to the order of the Weyl group we give a counting formula for the number of conjugacy classes of elements $x$ of order $N$ in $\mathfrak{G}$ with the property that the $N$-cyclotonic field when viewed as a Galois extension of the field of characters of $x$ has Galois group containing a fixed chosen cyclic group $\mathscr{G}$. The case $\mathscr{G}=\{1\}$ recovers a formula, due to Djokovic, which counts the number of conjugacy classes of elements of order dividing $N$ in $\mathfrak{G}$.
\end{abstract}

\section{INTRODUCTION}

In this paper we derive polynomial formulas for the number of conjugacy classes of elements of order $N$ coprime with the order of the Weyl group $W$ of a compact connected simple Lie group whose character values generate an extension of $\mathbb{Q}$ contained in certain number fields.

This result generalizes that of [DP] ([PW]) where the case $N=p^{K} \quad(N=p$, respectively) was treated. It also generalizes Djoković [Djk1].

Let $\mathfrak{G}$ be a (real) compact connected simple Lie group. As in [DP] and [PW] we define a lattice $\Gamma$ corresponding to a fixed maximal torus $\mathfrak{T}$ of $\mathfrak{G}$ via the following short exact sequence

$$
0 \rightarrow \Gamma \rightarrow \mathfrak{t} \stackrel{\exp 2 \pi i(\cdot)}{\longrightarrow} \mathfrak{T} \rightarrow 1
$$

where $\mathfrak{t}=i \mathfrak{t}^{\prime}$, with $\mathfrak{t}^{\prime}$ the Lie algebra of $\mathfrak{T}$, and $i=\sqrt{-1}$.

The Weyl group $W$ of $\mathfrak{G}$ acts on the lattice $\Gamma$ and hence on $V=\mathbb{C} \otimes_{\mathbf{z}} \Gamma$. Given an element $w \in W$, the multiplicity of the $d$ th root of unity $\zeta_{d}:=e^{2 \pi i / d}$ as an eigenvalue of $w$ in $V$ is denoted by

$$
f_{d}(w) .
$$

Received by the editors June 1, 1992 and, in revised form, October 5, 1992.

1991 Mathematics Subject Classification. Primary 22E15, 17B20.

The authors acknowledge the support of the CRM in Montreal and of NSERC Canada.

Work of the first author was supported in part by the Natural Sciences and Engineering Research Council of Canada and by the funds FCAR du Québec.

Research of the second author supported in part by the Natural Sciences and Engineering Research Council of Canada. 
(If $\zeta_{d}$ is not an eigenvalue of $w, f_{d}(w)$ is defined to be 0 .) Note that $d \mid m_{i}+1$ for some $1 \leq i \leq l$, where $m_{1}, \ldots, m_{l}$ are the exponents of $\mathfrak{G}$. In particular [Spg, Theorem 3.4, PW, Theorem 2], $1 \leq d \leq h$ where $h$ is the Coxeter number of $\mathfrak{G}$.

We define a "universal" polynomial $G\left(t_{1}, \ldots, t_{h}\right) \in \mathbb{Q}\left[t_{1}, \ldots, t_{h}\right]$ (with $h$ the Coxeter number of $\mathfrak{G}$ ) as follows:

$$
G\left(t_{1}, \ldots, t_{h}\right):=\frac{1}{|W|} \sum_{k_{1}, \ldots, k_{h} \in \mathbb{N}} a_{k_{1}, \ldots, k_{h}} t_{1}^{k_{1}} \cdots t_{h}^{k_{h}}
$$

where $a_{k_{1}, \ldots, k_{h}}$ is the number of elements of $W$ that have $\zeta_{i}$ as an eigenvalue of multiplicity $k_{i}$, for all $1 \leq i \leq h$. Equivalently, $G$ is given by the average sum

$$
G\left(t_{1}, \ldots, t_{h}\right):=\frac{1}{|W|} \sum_{w \in W} t_{1}^{f_{1}(w)} \cdots t_{h}^{f_{h}(w)} .
$$

We consider elements $x$ of finite order of $\mathfrak{G}$. If $x \in \mathfrak{G}$ has order $N$, then the character value $\operatorname{tr}_{V}(\rho(x))$ of any finite dimensional (complex) representation $(\rho, V)$ of $\mathfrak{G}$ is contained in the cyclotomic field $\mathbb{Q}\left(\zeta_{N}\right)$ where $\zeta_{N}=e^{2 \pi i / N}$. The subfield of $\mathbb{Q}\left(\zeta_{N}\right)$ generated by all such character values is called the field of characters of $x$ and is denoted by $A(x)$. As in [DP], we then define the Galois group of $x$ by

$$
\operatorname{Gal}(x):=\operatorname{Gal}\left(\mathbb{Q}\left(\zeta_{N}\right) / A(x)\right)
$$

which we will view as a subgroup of $(\mathbb{Z} / N \mathbb{Z})^{*}$ by means of the natural description of $\operatorname{Gal}\left(\mathbb{Q}\left(\zeta_{N}\right) / \mathbb{Q}\right)$.

We fix a positive integer $N$ with prime factorization

$$
N=p_{1}^{K_{1}} \cdots p_{s}^{K_{s}}
$$

where $p_{1}, \ldots, p_{s}$ are distinct primes not dividing the order of the Weyl group $W$ of $\mathfrak{G}$. We fix a cyclic subgroup $\mathscr{C}$ of $(\mathbb{Z} / N \mathbb{Z})^{*}$ as well as a generator

$$
a \in \mathscr{C}
$$

and we set, for $1 \leq i \leq s$,

$$
n_{i}=\operatorname{ord}\left(a_{i}\right)
$$

in $\left(\mathbb{Z} / p_{i}^{K_{i}} \mathbb{Z}\right)^{*}$, where $a_{i}$ is the image of $a$ under the canonical projection $(\mathbb{Z} / N \mathbb{Z})^{*} \rightarrow\left(\mathbb{Z} / p_{i}^{K_{i}} \mathbb{Z}\right)^{*}$.

The main result of this paper is the following theorem.

Theorem. Notation as above. The number of conjugacy classes of elements of $\mathfrak{G}$ of order $N$ with $(a) \subseteq \operatorname{Gal}(x)$ is given by

$$
\sum_{\varepsilon=\left(\varepsilon_{1}, \ldots, \varepsilon_{s}\right) \in(\mathbb{Z} / 2 \mathbb{Z})^{s}}(-1)^{|\varepsilon|} G\left(q_{1}(\varepsilon), \ldots, q_{h}(\varepsilon)\right)
$$

where $q_{j}(\varepsilon)=\prod_{i: n_{i}=j} p_{i}^{\left(K_{i}-\varepsilon_{i}\right)}$ and $|\varepsilon|=\sum_{i=1}^{s} \varepsilon_{i}$. 


\section{Counting Weyl group orbits}

In this section we recall the basic approach adopted in [DP, Pzl1, PW].

The Weyl group $W$ of $\mathfrak{G}$ acts on the lattice $\Gamma$ and hence on the module $\Gamma / N \Gamma$, for any positive integer $N$. Observe that $W$ also acts on the set of reduced elements of $\Gamma / N \Gamma$; i.e., those elements of order $N$.

Proposition 1. (a) Let $x$ be an elemenet of $\mathfrak{G}$ of order $N$. Let $\operatorname{Gal}(x)$ be as in (5). Then $|\mathrm{Gal}(x)|$ divides $(\phi(N),|W|)$. In particular, $|\mathrm{Gal}(x)|$ divides $\prod_{i=1}^{s}\left(p_{i}-1\right)$. Moreover, for any $g \in \mathrm{Gal}(x)$, the order $\operatorname{ord}(g)$ of $g$ divides the exponent $e(W)$ of $W$ (i.e., the least common multiple of the orders of elements of $W$ ).

(b) Let $\mathscr{G}$ be a subgroup of $\operatorname{Gal}\left(\mathbb{Q}\left(\zeta_{N}\right) / \mathbb{Q}\right)$ (with $|\mathscr{G}|$ dividing $\prod_{i=1}^{s}\left(p_{i}-1\right)$ ) and $\{a(\alpha)\}_{\alpha \in I}$ be a family of generators of $\mathscr{G}$ under the canonical isomorphism $\operatorname{Gal}\left(\mathbb{Q}\left(\zeta_{N}\right) / \mathbb{Q}\right) \approx(\mathbb{Z} / N \mathbb{Z})^{*}$. The conjugacy classes of elements $x$ of $\mathfrak{G}$ of order $N$ such that $\mathscr{G} \subseteq \operatorname{Gal}(x)$ are in bijection with $W$-orbits of reduced elements $z$ of $\Gamma / N \Gamma$ such that, for any $\alpha \in I$, there exists some $w(\alpha) \in W$ for which $w(\alpha) z=a(\alpha) z$.

Proof. From [DP, Proposition 1(b)], if $x$ corresponds to $z \in \Gamma / N \Gamma$, then there is an isomorphism $W_{z}^{e} / W_{z} \stackrel{\approx}{\rightarrow} \mathrm{Gal}(x)$ where $W_{z} \subseteq W_{z}^{e}$ are certain subgroups of $W$. Hence, $|\mathrm{Gal}(x)|$ divides $|W|$. Of course $|\mathrm{Gal}(x)|$ divides also $\left|(\mathbb{Z} / N \mathbb{Z})^{*}\right|=\phi(N)=\prod_{i=1}^{s} \rho^{K_{i}-1}\left(p_{i}-1\right)$ (from $\left.(6)\right)$. Since $(N,|W|)=1$ we conclude that $|\mathrm{Gal}(x)|$ divides $\prod_{i=1}^{s}\left(p_{i}-1\right)$. Finally, $e(W)$ kills any element of $W$, hence, of $W_{z}^{e} / W_{z}$, and hence, of $\operatorname{Gal}(x)$. Thus $\operatorname{ord}(g) \mid e(W)$. This proves part (a).

[Pzl1] shows the existence of a 1-1 correspondence between conjugacy classes of elements of $\mathfrak{G}$ of order $N$ and $W$-orbits of reduced elements of $\Gamma / N \Gamma$. Moreover, from [DP, Proposition 1(c)] under this bijection and the canonical isomorphism $\operatorname{Gal}\left(\mathbb{Q}\left(\zeta_{N}\right) / \mathbb{Q}\right) \approx(\mathbb{Z} / N \mathbb{Z})^{*}, \operatorname{Gal}(x)$ corresponds to $\operatorname{Gal}(z):=$ $\left\{k \in(\mathbb{Z} / N \mathbb{Z})^{*}: w z=k z\right.$ for some $\left.w \in W\right\}$. Thus, with the notation as in the statement of the proposition, $\mathscr{G} \subseteq \operatorname{Gal}(x)$ if and only if $a(\alpha) \in \operatorname{Gal}(z)$, for each $\alpha \in I$. This proves part (b) of the proposition.

For the remaining part of this section we consider the case of a cyclic group $\mathscr{C}=(a) \subseteq(\mathbb{Z} / N \mathbb{Z})^{*}$ as in $(7)$, and we denote by

$$
a_{i} \quad(1 \leq i \leq s)
$$

the image of $a$ under the canonical homomorphism $(\mathbb{Z} / N \mathbb{Z})^{*} \approx \prod_{i=1}^{s}\left(\mathbb{Z} / p_{i}^{K_{i}} \mathbb{Z}\right)^{*}$ $\rightarrow\left(\mathbb{Z} / p_{i}^{K_{i}} \mathbb{Z}\right)^{*}$.

Consider the torsion $\mathbb{Z} W$-modules

$$
M=\Gamma / N \Gamma, \quad M_{i}=\Gamma / p_{i}^{K_{i}} \Gamma \quad(1 \leq i \leq s) .
$$

The canonical projections $\pi_{i}: M \rightarrow M_{i}(1 \leq i \leq s)$ yield an isomorphism of $\mathbb{Z} W$-modules

$$
\pi:=\bigoplus_{i=1}^{s} \pi_{i}: M \stackrel{s}{\underset{\sim}{\rightarrow}} \bigoplus_{i=1} M_{i}
$$


Note that, if $z \in M$ is mapped to $\left(z_{i}\right)_{i=1}^{s}$ under this isomorphism then, given any $w \in W$, we have the equivalence

$$
w z=a z \text { if and only if } w z_{i}=a_{i} z_{i} \text { for all } 1 \leq i \leq s .
$$

Keeping the notation above, we define the sets

$$
\begin{aligned}
Y(a, w) & :=\{z \in M: z \text { is reduced and } w z=a z\} \\
Y\left(a_{i}, w\right) & :=\left\{z_{i} \in M_{i}: z_{i} \text { is reduced and } w z_{i}=a_{i} z_{i}\right\} .
\end{aligned}
$$

Lemma 1. Notation as in (9)-(12). The isomorphism $\pi$ induces a set theoretical bijection

$$
Y(a, w) \leftrightarrow \stackrel{s}{\times} Y\left(a_{i}, w\right) .
$$

Proof. This follows immediately from (9)-(11).

Lemma 2. Notation as in (9)-(12). The number of conjugacy classes of elements of $\mathfrak{G}$ of order $N$ with $(a) \subseteq \operatorname{Gal}(x)$ is given by

$$
\frac{1}{|W|} \sum_{w \in W} \prod_{i=1}^{s}\left|Y\left(a_{i}, w\right)\right|
$$

Proof. The argument found in the proof of [DP, Lemma 1] yields the identity

$$
\sum_{w \in W}|Y(a, w)|=|W| c
$$

where $c$ is the number of $W$-orbits of reduced elements of $M$ for which $w z=a z$ for some $w \in W$. Now use Proposition 1(b) and Lemma 1.

\section{Proof of The THeOREM}

In this section we consider, as in (6), a positive integer $N=\prod_{i=1}^{s} p_{i}^{K_{i}}$ relatively prime with $|W|$. As in (7), we fix a cyclic subgroup $\mathscr{C}$ with generator $a \in(\mathbb{Z} / N \mathbb{Z})^{*}$; we let $n_{i}, 1 \leq i \leq s$, be as in (8).

Proposition 2. The number of conjugacy classes of elements of $\mathfrak{G}$ of order $N$ with $\mathscr{C}=(a) \subseteq \operatorname{Gal}(x)$ is given by

$$
\frac{1}{|W|} \sum_{w \in W} \prod_{i=1}^{s}\left(p_{i}^{K_{i} f_{n_{i}}(w)}-p_{i}^{\left(K_{i}-1\right) f_{n_{i}}(w)}\right)
$$

where the $f_{n_{i}}(w)$ are as in (2).

Proof. This is an immediate consequence of Lemma 2 and [DP, Lemma 2] $\left(\left|Y\left(a_{i}, w\right)\right|=p_{i}^{K_{i} f_{n_{i}}(w)}-p_{i}^{\left(K_{i}-1\right) f_{n_{i}}(w)}\right.$, since $n_{i}$ is the order of $a_{i}$ in $\left.\left(\mathbb{Z} / p_{i}^{K_{i}} \mathbb{Z}\right)^{*}\right)$.

We can reformulate Proposition 2 in terms of the polynomial $G$ defined in (3) and (4). 
Proof of the Theorem. Notation as above. We have the equalities

$$
\begin{aligned}
& \prod_{i=1}^{s}\left(p_{i}^{K_{i} f_{n_{i}}(w)}-p_{i}^{\left(K_{i}-1\right) f_{n_{i}}(w)}\right) \\
& =\sum_{\varepsilon=\left(\varepsilon_{1}, \ldots, \varepsilon_{s}\right) \in(\mathbb{Z} / 2 \mathbb{Z})^{s}}(-1)^{|\varepsilon|} p_{1}^{\left(K_{1}-\varepsilon_{1}\right) f_{n_{1}}(w)} \cdots p_{s}^{\left(K_{s}-\varepsilon_{s}\right) f_{n_{s}}(w)} \\
& \quad=\sum_{\varepsilon=\left(\varepsilon_{1}, \ldots, \varepsilon_{s}\right) \in(\mathbb{Z} / 2 \mathbb{Z})^{s}}(-1)^{|\varepsilon|} \prod_{j=1}^{n}\left(\prod_{i: n_{i}=j} p_{i}^{\left(K_{i}-\varepsilon_{i}\right)}\right)^{f_{j}(w)} \\
& \quad=\sum_{\varepsilon=\left(\varepsilon_{1}, \ldots, \varepsilon_{s}\right) \in(\mathbb{Z} / 2 \mathbb{Z})^{s}}(-1)^{|\varepsilon|} q_{1}(\varepsilon)^{f_{1}(w)} \cdots q_{h}(\varepsilon)^{f_{h}(w)} .
\end{aligned}
$$

The theorem now follows from Proposition 2.

Corollary 1 (Djoković [Djk1]). Let $N$ be coprime with $|W|$. The number of conjugacy classes of elements of $\mathfrak{G}$ of exponent $N$ is

$$
\nu(N)=\prod_{i=1}^{l} \frac{m_{i}+N}{m_{i}+1}
$$

where $m_{1} \leq m_{2} \leq \cdots \leq m_{l}$ are the exponents of the Weyl group $W$ of $\mathfrak{G}$.

Proof. We first evaluate the number of conjugacy classes of elements of $\mathfrak{G}$ of order $N$ with $(1) \subseteq \operatorname{Gal}(x)$ by looking at the particular case $n_{1}=n_{2}=\cdots=$ $n_{s}=1$ in (8). From the Theorem this number equals

$$
\sum_{\varepsilon=\left(\varepsilon_{1}, \ldots, \varepsilon_{s}\right) \in(\mathbb{Z} / 2 \mathbb{Z})^{s}}(-1)^{|\varepsilon|} G\left(q_{1}(\varepsilon), \ldots, q_{h}(\varepsilon)\right)
$$

where here $q_{1}(\varepsilon)=\prod_{i=1}^{s} p^{\left(K_{i}-\varepsilon_{i}\right)}, q_{j}(\varepsilon)=1 \quad(j \neq 1)$, and $|\varepsilon|=\sum_{i=1}^{s} \varepsilon_{i}$.

For a fixed $\varepsilon$, we have

$$
\begin{aligned}
G\left(q_{1}(\varepsilon), \ldots, q_{h}(\varepsilon)\right) & =\frac{1}{|W|} \sum_{k_{1}, \ldots, k_{h} \in \mathbb{N}} a_{k_{1}, \ldots, k_{h}} q_{1}(\varepsilon)^{k_{1}} \\
& =\frac{1}{|W|} \sum_{k_{1}}\left(\sum_{k_{2}, \ldots, k_{h}} a_{k_{1}, \ldots, k_{h}}\right) q_{1}(\varepsilon)^{k_{1}} .
\end{aligned}
$$

Note that $\sum_{k_{2}, \ldots, k_{h}} a_{k_{1}, \ldots, k_{h}}$ is the number of elements $w$ of $W$ with 1 as an eigenvalue of multiplicity $k_{1}$, which we will denote $s_{k_{1}}(1)$ (as in [PW]). Thus,

$$
G\left(q_{1}(\varepsilon), \ldots, q_{h}(\varepsilon)\right)=\frac{1}{|W|} \sum_{k_{1}} s_{k_{1}}(1) q_{1}(\varepsilon)^{k_{1}} .
$$

But, from [Slm], the right-hand side of this equality is given by $\prod_{i=1}^{l}\left(m_{i}+q_{1}(\varepsilon)\right) /\left(m_{i}+1\right)$ where $m_{1}, \ldots, m_{l}$ are the exponents of $W$.

As an intermediate step we therefore have the following

Corollary 2. The number of conjugacy classes of elements of $\mathfrak{G}$ of order $N$ is given by

$$
\sum_{\varepsilon=\left(\varepsilon_{1}, \ldots, \varepsilon_{s}\right) \in(\mathbb{Z} / 2 \mathbb{Z})^{s}}(-1)^{|\varepsilon|} \nu\left(q_{1}(\varepsilon)\right)
$$

where $\nu(t):=\prod_{i=1}^{l}\left(m_{i}+t\right) /\left(m_{i}+1\right)$. 
We now finish the proof of Corollary 1. Assume Djoković's formula is true for any $M<N$ (it is obviously true for $N=1$ ). Then, using an "inclusionexclusion" argument, the number of conjugacy classes of elements of exponent $N$ but order less than $N$ is easily seen to be

$$
\sum_{(0, \ldots, 0) \neq \varepsilon \in(\mathbb{Z} / 2 \mathbb{Z})^{s}}(-1)^{|\varepsilon|-1} \nu\left(q_{1}(\varepsilon)\right) .
$$

Combining (13) and (14), we conclude that the total number of conjugacy classes of elements of exponent $N$ is equal to $\nu\left(q_{1}(0)\right)$, which is $\nu(N)$, as was to be shown.

Next, we show how [DP, Theorem 1] follows from the Theorem.

We fix a prime number $p$ not dividing $|W|$, and an integer $K \geq 1$. We set $N=p^{K}$. So, adopting the notation of (6), we have $s=1$. We fix a subgroup of $\operatorname{Gal}\left(\mathbb{Q}\left(\zeta_{N}\right) / \mathbb{Q}\right) \approx\left(\mathbb{Z} / p^{K} Z\right)^{*}$, necessarily cyclic, say $(a)$. We set $n=\operatorname{ord}(a)$ as in (8). So, here, $n_{1}=n$.

If $x$ is any element of $\mathfrak{G}$ of order $N=p^{K}$, then its $\operatorname{Galois} \operatorname{group} \operatorname{Gal}(x)$ is a subgroup of the cyclic group $\operatorname{Gal}\left(\mathbb{Q}\left(\zeta_{N}\right) / \mathbb{Q}\right)$, and hence is determined by its order, called the depth of $x$.

[DP, Theorem 1(a)] counts the number of conjugacy classes of elements of order $p^{K}$, with depth divisible by $n$, i.e., in the present context, with $(a) \subseteq$ $\mathrm{Gal}(x)$. In view of Proposition 1(a) (or [DP, Proposition 2(a)]), we may assume that $n \mid \operatorname{gcd}(p-1, e(w))$, as in the hypothesis of [DP, Theorem 1(a)].

In [DP, PW], the polynomials used to do this counting, are

$$
A_{n}(t)=-1+\prod_{i \in I(n)} \frac{m_{i}+t}{m_{i}+1}
$$

where $m_{1} \leq m_{2} \leq \cdots \leq m_{l}$ are the exponents of the Weyl group $W$ of $\mathfrak{G}$, and where $I(n):=\left\{1 \leq i \leq l: n\right.$ divides $\left.m_{i}+1\right\}$. But from [PW, Theorem 2], we have

$$
A_{n}(t)=\frac{1}{|W|} \sum_{w \in W} t^{f_{n}(w)}-1,
$$

i.e.,

$$
A_{n}(t)=G\left(t_{1}, \ldots, t_{h}\right)-1
$$

with

$$
\begin{array}{ll}
t_{n}:=t & \text { if } 1 \leq n \leq h \\
t_{j}:=1 & \text { for any } 1 \leq j \neq n \leq h .
\end{array}
$$

Finally, the expression

$$
\sum_{\varepsilon \in(\mathbb{Z} / 2 \mathbb{Z})^{s}}(-1)^{|\varepsilon|} G\left(q_{1}(\varepsilon), \ldots, q_{h}(\varepsilon)\right)
$$

of the Theorem, now reads as

$$
\sum_{\varepsilon=0,1}(-1)^{\varepsilon} G\left(q_{1}(\varepsilon), \ldots, q_{h}(\varepsilon)\right)
$$


where

$$
\begin{array}{ll}
q_{n}(\varepsilon)=p^{K-\varepsilon} & \text { if } 1 \leq n \leq h, \\
q_{j}(\varepsilon)=1 & \text { for any } 1 \leq j \leq h, j \neq n
\end{array}
$$

From (15), we rewrite (16) as $A_{n}\left(p^{K}\right)-A_{n}\left(p^{K-1}\right)$. All together, we have derived the following result from the Theorem.

Corollary 3 [DP, Theorem 1]. Let $p$ be a prime number not dividing $|W|$. Let $n \mid \operatorname{gcd}(p-1), e(w))$. The number of conjugacy classes of elements of $\mathfrak{G}$ of order $p^{K}$, with $p^{K} \geq 1$, and depth divisible by $n$ is $A_{n}\left(p^{K}\right)-A_{n}\left(p^{K-1}\right)$.

N.b. We have derived Theorem 1 of [DP] from the Theorem above, but note that the proof of the present theorem makes use of the crucial lemmas and propositions in [DP].

\section{A REMARK ON COMPUTATIONS}

The polynomial $G\left(t_{1}, \ldots, t_{h}\right)$ for a particular Weyl group can explicitly be computed using the work of Carter [Ctr], where conjugacy classes of the Weyl group $W$ of $\mathfrak{G}$ are described by certain graphs (or Dynkin diagrams) denoted in [Ctr] by $\Gamma$ (not to be confused with our notation in (1)!) (see [Ctr, pp. 2431, for classical groups and pp. 31-34 and pp. 48-58 for the exceptional ones]). Also, one can find in [Ctr], for each graph $\Gamma$, the cardinality $\left|\operatorname{ccl}_{W}(w)\right|$ of the conjugacy class of the corresponding element $w$ of $W$ (see [Ctr, pp. 34-45, 48-58]).

Finally, the characteristic polynomial $p_{w}(t)$ of an element $w$ of $W$ is computed from a graph $\Gamma$ representing its conjugacy class, using [Ctr, Table 3, p. 23], via a straightforward algorithm [Ctr, pp. 19-24]. Knowing $p_{w}(t)$, one can then deduce easily the multiplicity $f_{j}(w)$ of $\zeta_{j}$ as an eigenvalue of $w$, for each $1 \leq j \leq h$.

We then use the expression

$$
G\left(t_{1}, \ldots, t_{h}\right)=\frac{1}{|W|} \sum_{\Gamma}\left|\operatorname{ccl}_{W}(w)\right| t_{1}^{f_{1}(w)} \cdots t_{h}^{f_{h}(w)},
$$

which follows from (4) using the fact that the monomials $t_{1}^{f_{1}(w)} \cdots t_{h}^{f_{h}(w)}$ are invariant under conjugation in $W$.

\section{REFERENCES}

[Ctr] R. W. Carter, Conjugacy classes in the Weyl group, Compositio Math. 25 (1972), 1-59.

[DP] F. Destrempes and A. Pianzola, Elements of compact connected simple Lie groups with prime power order and given field of characters, Geom. Dedicata 45 (1993), 225-235.

[Djk1] D. Djoković, On conjugacy classes of elements of finite order in compact or complex semisimple Lie groups, Proc. Amer. Math. Soc. 80 (1980), 181-184.

[Djk2] __ On the conjugacy classes of elements of finite order in complex semisimple Lie groups, J. Pure Appl. Algebra 35 (1985), 1-13.

[MPt] R. V. Moody and J. Patera, Characters of elements of finite order in Lie groups, SIAM J. Algebra Discrete Methods 5 (1984), 359-383.

[Pzl1] A. Pianzola, On the arithmetic of the representation ring and elements of finite order in Lie groups, J. Algebra 108 (1987), 1-33. 
[Pz12] _ On the rationality and regularity of certain elements of finite order in Lie groups, $\mathrm{J}$. Reine Angew. Math. 377 (1987), 40-48.

[PW] A. Pianzola and A. Weiss, The rationality of elements of prime order in compact connected simple Lie group, J. Algebra 144 (1991), 510-521.

[Slm] L. Solomon, Invariants of finite reflection groups, Nagoya Math. J. 22 (1963), 57-64.

[Spg] T. A. Springer, Regular elements of finite reflection groups, Invent. Math. 25 (1974), 159-198.

CRM, Université de Montréal CP6128-A, Montréal, Québec, Canada H3C 3J7

Current address: Department of Mathematics, University of Toronto, Toronto, Ontario, Canada M5S 1A1

E-mail address: francois@math.toronto.edu

Department of Mathematics, University of Alberta, Edmonton, Alberta, Canada T6G 2G1

E-mail address: apianzol@vega.math.ualberta.ca 